\title{
Retinoblastoma bilateral de aparecimento tardio: relato de caso
}

\author{
Late presentation of bilateral retinoblastoma: case report
}

\author{
Maria Cecília Santos Cavalcanti Melo ${ }^{1}$ \\ Liana Maria Vieira de Oliveira Ventura ${ }^{2}$ \\ Clélia Maria Erwenne ${ }^{3}$ \\ Ana Lúcia Andrade Lima Arcoverde ${ }^{4}$
}

\begin{tabular}{|l|}
\hline RESUMO \\
\hline É relatado um caso de retinoblastoma de aparecimento tardio, com carac- \\
terísticas pouco freqüentes, com o objetivo de melhorar o controle da \\
afecção. Em 1993, SJMMF, nove meses, masculino, leucodermo, apresen- \\
tou tumor na retina esquerda. O paciente tinha história familiar de re- \\
tinoblastoma. A enucleação foi realizada, comprovando-se retinoblastoma. \\
Os exames para estadiamento sistêmico foram normais. O olho direito \\
evoluiu normal por dez anos. Em exame de rotina, foram constatadas três \\
lesões de retinoblastoma naretina nasal. Foi feita termoterapia transpupilar, \\
com laser de diodo de 810 nm. Após 30 dias, as lesões regrediram. Após \\
60 dias houve recidiva na borda da lesão, onde foi realizada crioterapia \\
transescleral, com regressão do tumor por seis meses. Durante o controle, \\
observaram-se condensações próximas à lesão tumoral atrófica (sementes \\
vítreas). Foi feito braquiterapia com Iodo125, havendo desaparecimento \\
das mesmas após 30 dias. Novas sementes surgiram três meses pós- \\
braquiterapia, depositadas na superfície retiniana, sendo tratadas com \\
crioterapia transescleral e termoterapia transpupilar, havendo regressão. \\
O paciente evoluiu com nova semente vítrea após seis meses, a qual, após \\
depositar-se na superfície da retina, foi tratada com termoterapia trans- \\
pupilar. Está em seguimento há 38 meses desde o aparecimento do tumor \\
bilateral, mantendo acuidade visual de $20 / 20$ e exames clínicos normais. \\
Considera-se importante este caso pela pouca freqüência de aparecimento \\
da doença nesta idade. Julga-se necessário o alerta para os casos de \\
retinoblastoma já considerados curados. \\
\hline
\end{tabular}

Descritores: Retinoblastoma/epidemiologia; Enucleação ocular; Lasers; Hipertermia induzida; Humanos; Masculino; Lactente; Relatos de casos [Tipo de publicação]

\section{INTRODUÇÃOO}

Embora o retinoblastoma seja um tumor relativamente incomum, ocorre numa proporção de 1:15.000 a 1:20.000 nascidos vivos ${ }^{(1)}$. Nos EUA, aproximadamente 300 crianças e adolescentes menores que 20 anos de idade são diagnosticadas com a doença a cada ano ${ }^{(2)}$. No Brasil, a notificação de dados sobre a incidência deste tumor é ainda desconhecida, havendo relatos de variações entre diversas regiões, sendo descritos 5,9 casos por milhão de habitantes em São Paulo(3).

O retinoblastoma tem origem neuroectodérmica, apresentando-se em sua forma hereditária em $40 \%$ dos casos, com herança autossômica dominante, ligada à mutação no braço longo do cromossomo 13. Clinicamente é evidenciado de forma bilateral, uni ou multifocal. Em $60 \%$ dos casos, na sua forma esporádica, o retinoblastoma é geralmente unilateral e unifocal. Em 5 a 15\% 
dos casos bilaterais pode-se encontrar o tumor desenvolvendo-se na glândula pineal (pinealoblastoma ou retinoblastoma trilateral $)^{(4-8)}$.

A maioria dos casos ocorre em crianças bastante jovens, com $63 \%$ abaixo de dois anos de idade e $95 \%$ antes dos cinco anos ${ }^{(2)}$. Após essa faixa etária, o aparecimento de retinoblastoma é raro, observando-se descrição de poucos casos na literatura ${ }^{(4-8)}$.

Este estudo tem por objetivo relatar um caso de retinoblastoma caracteristicamente incomum em sua apresentação clínica e evolução, bem como discutir a conduta terapêutica instituída em um serviço de referência em oncologia ocular, assim como estimular o controle rigoroso na condução desta doença.

\section{RELATO DO CASO}

Em 1993, SJMMF, nove meses de idade, gênero masculino, leucodermo, procedente da zona rural de Alagoas, apresentou desvio no olho esquerdo, percebido pelo seu genitor. Foi avaliado por oftalmologista, sendo constatado a presença de um "tumor na retina".

O pai apresentou história de desvio ocular por lesão na retina desde infância (provável retinoblastoma de regressão espontânea). O primo paterno apresentou história de enucleação em um dos olhos por tumor maligno. Negava consangüinidade.

Na ocasião, submeteu-se a enucleação do olho esquerdo, sem intercorrências. Por problemas técnicos durante a preparação do espécime para análise anátomo-patológica (carbonização do material), houve dificuldades para a interpretação das lâminas, onde apenas se observou células com formação em rosetas, não sendo possível a análise do grau de diferenciação e extensão do tumor ao nervo óptico. O laudo do patologista limitou-se a compatibilidade com retinoblastoma, sem possibilidade de estadiamento. O caso evoluiu com a realização de exames completos (tomografia computadorizada e ressonância nuclear magnética de crânio e órbita, análise do líquido cefalorraquidiano, mielograma) para estadiamento clínico, realizados com oncologista pediátrico, os quais foram normais. Após análise com equipe multidisciplinar, o caso foi considerado como retinoblastoma intra-ocular unilateral, não sendo indicado o tratamento com quimioterapia adicional. $\mathrm{O}$ paciente foi acompanhado com exames periódicos de controle sistêmico e oftalmológico, que se mantiveram normais por 10 anos.

Em julho de 2003, compareceu ao oftalmologista para exame anual, sem queixas, com acuidade visual sem correção de 20/20 e à biomicroscopia, segmento anterior sem anormalidades.

Ao mapeamento da retina evidenciaram-se três lesões esbranquiçadas < 1 diâmetro de disco (DD), seguindo arcada nasal inferior com nervo óptico, mácula e vítreo sem alterações. A suspeita diagnóstica foi retinoblastoma com aparecimento tardio. Realizou-se retinografia (Figuras 1 e 2) e angiografia fluoresceínica (Figura 3) para documentação e procedeu-se a aplicação de termoterapia transpupilar (TTT) com laser de

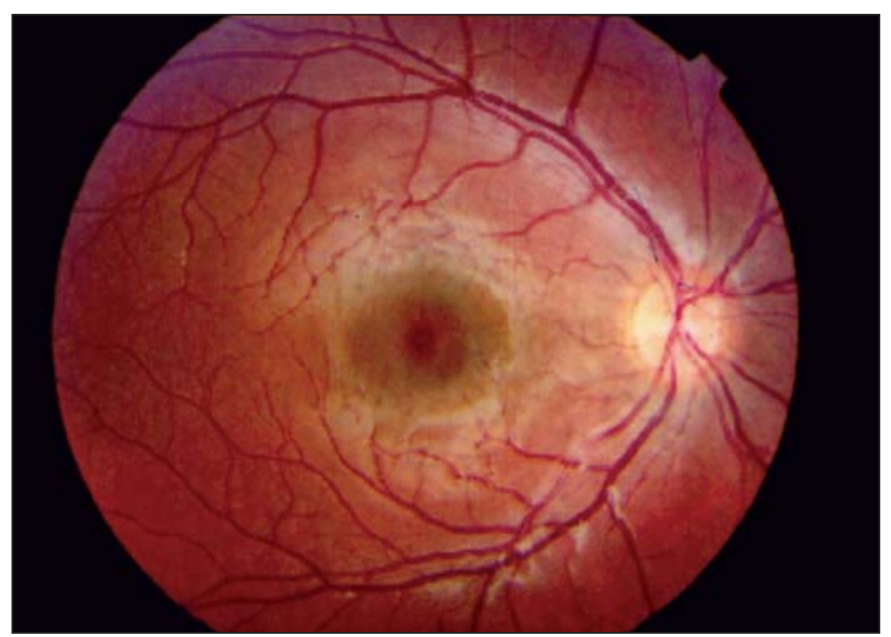

Figura 1 - Retinografia. Pólo posterior do olho direito sem alterações.

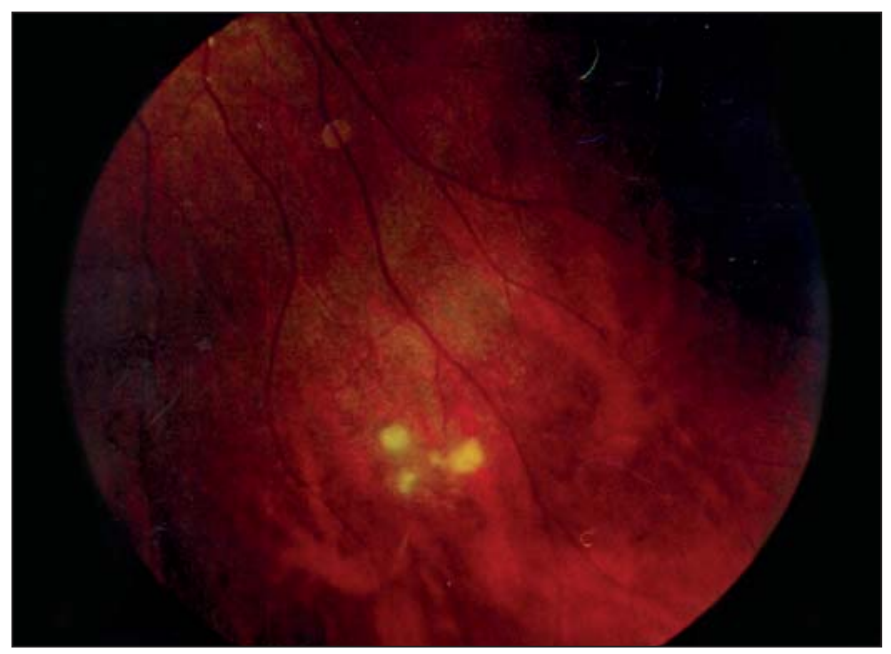

Figura 2 - Retinografia. Presença de três lesões esbranquiçadas, menores que $3 \mathrm{~mm}$ no equador retiniano nasal inferior do olho direito.

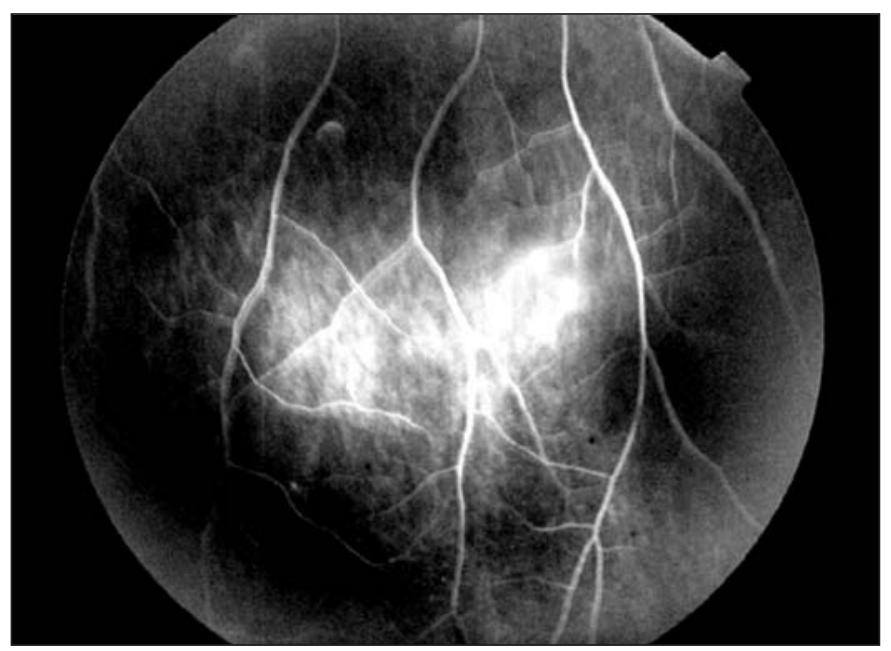

Figura 3 - Angiografia fluoresceínica - Hiperfluorescência seguindo as arcadas nasais inferiores correspondente à figura 1 
diodo de $810 \mathrm{~nm}$ por via oftalmoscópica indireta (mira de $3 \mathrm{~mm}$, duração de $60 \mathrm{~s}$ e potência de $400 \mathrm{~mW}$ ), em outro serviço. Após 30 dias deste procedimento o paciente retornou para controle, tendo sido observado que as lesões evoluíram com grau de regressão do tipo IV (plana e atrófica). Após dois meses, detectou-se recidiva do tumor na borda da lesão. Realizou-se uma sessão de crioterapia transescleral, obtendo-se controle por período de seis meses (Figuras 4 e 5).

Após sete meses da crioterapia detectou-se a presença de sementes tumorais vítreas, localizadas próximas à lesão tumoral atrófica (Figuras 6 e 7). Realizou-se ultra-sonografia ocular com cálculo da distância entre as sementes e a parede posterior do globo, tendo sido realizado braquiterapia com sementes de Iodo $^{125}$, incluindo-se a altura das sementes tumorais na área de tratamento. Após um mês de tratamento observou-se regressão das sementes vítreas.

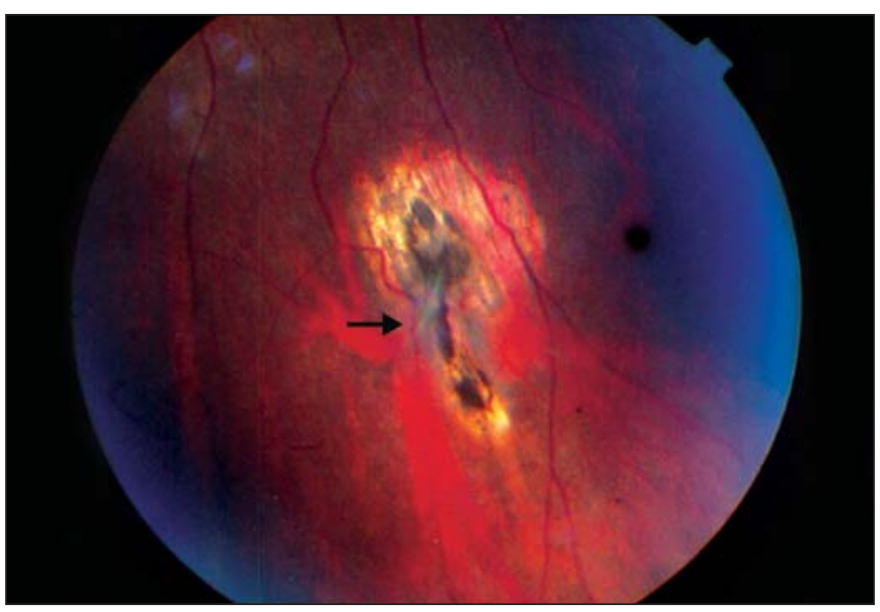

Figura 4 - Retinografia após realização de termoterapia transpupilar - Cicatriz plana, com áreas de atrofia do epitélio pigmentar da retina e áreas de hiperplasia pigmentar reacional. Observa-se condensação linear, esbranquiçada sobre a área, considerada como lesão residual pós tratamento.

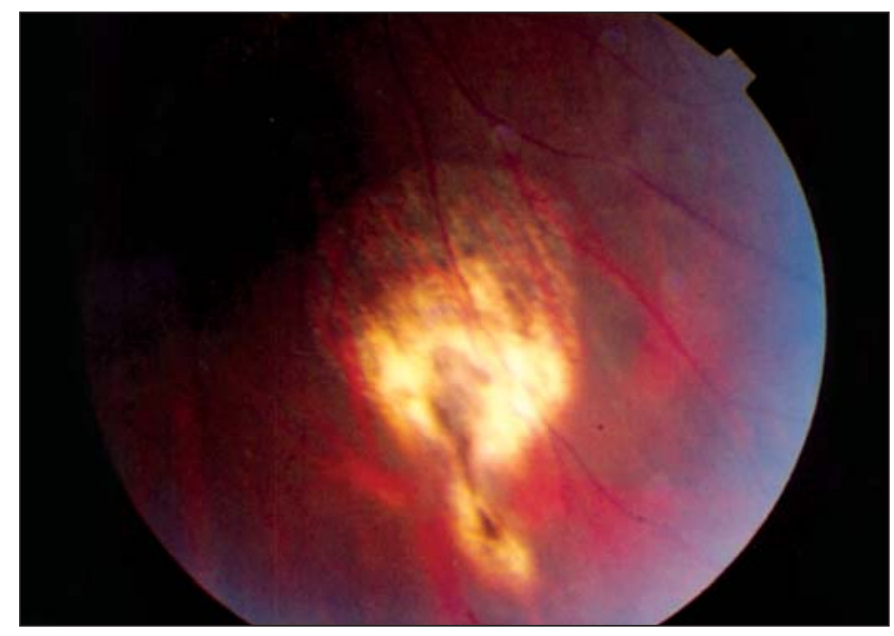

Figura 5 - Retinografia após crioterapia trasescleral - Ampliação da área cicatricial plana e atrófica sem lesões residuais
Com três meses de controle, surgiu uma nova condensação vítrea com toque retiniano próxima à borda da lesão atrófica, sendo esta considerada como nova semente tumoral.

Realizou-se ultra-sonografia ocular, detectando-se toque desta lesão na superfície da retina (Figura 8) e indicou-se aplicação de crioterapia com bom acesso ao ápice da lesão. Com 30 dias deste procedimento, notou-se subdivisão da condensação vítrea e base atrófica retiniana optando-se assim, por ampliação das bordas da lesão com TTT (Figura 9).

Por aproximadamente seis meses o paciente evoluiu com ausência completa dessas sementes e base tumoral atrófica. Em uma reavaliação, observou-se surgimento de outra condensação arredondada e flutuante, próxima à borda nasal da cicatriz atrófica que foi seguida quinzenalmente por meio de mapeamento de

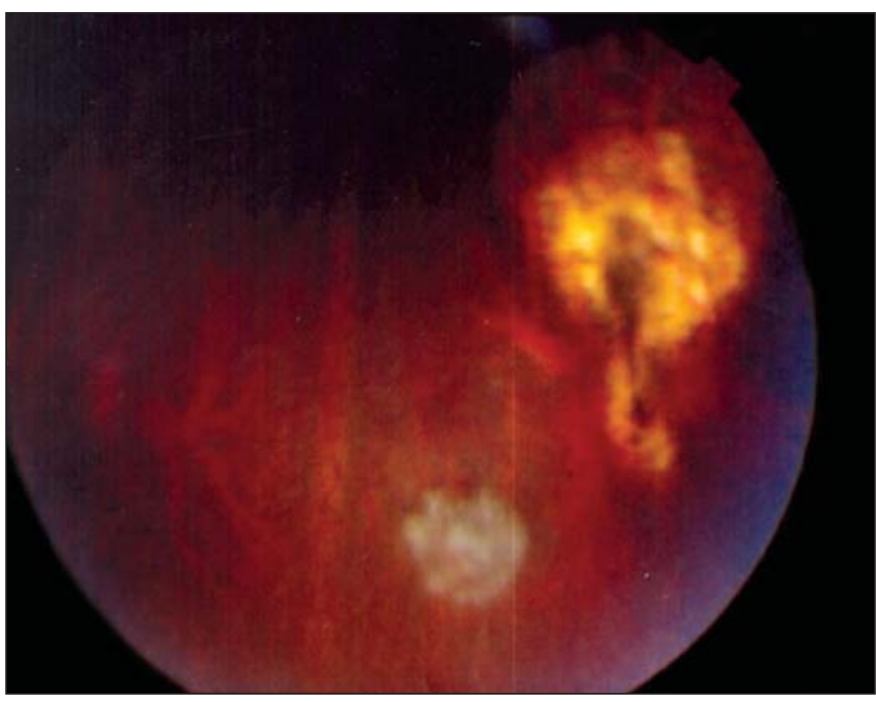

Figura 6 - Retinografia após 6 meses de follow-up - Área de cicatrização plana e atrófica estável. Presença de condensação circular, com 1 diâmetro de disco, esbranquiçada, flutuante em cavidade vítrea, próxima à lesão primária

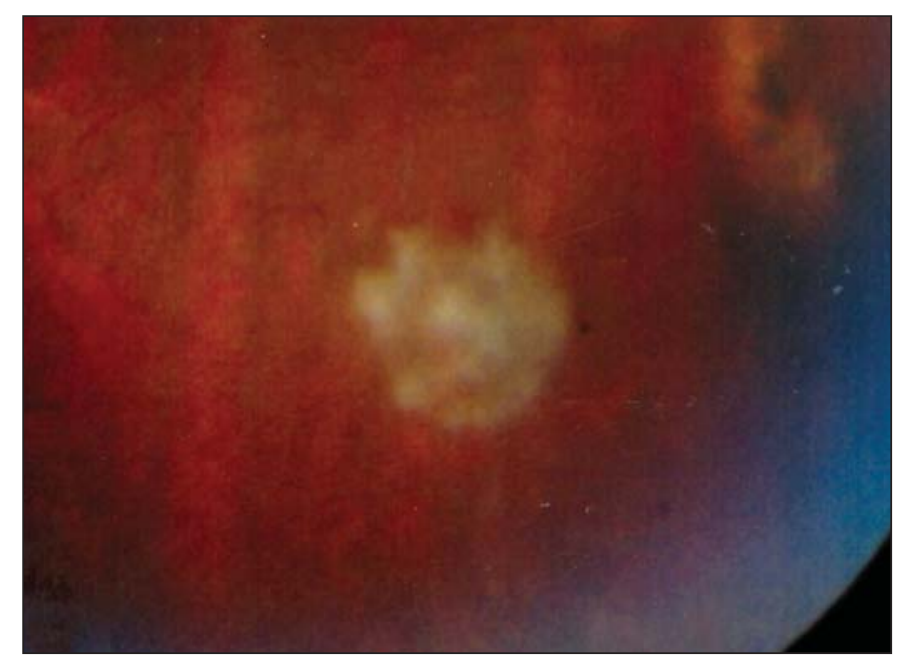

Figura 7 - Retinografia - Condensação flutuante antes da realização de braquiterapia 


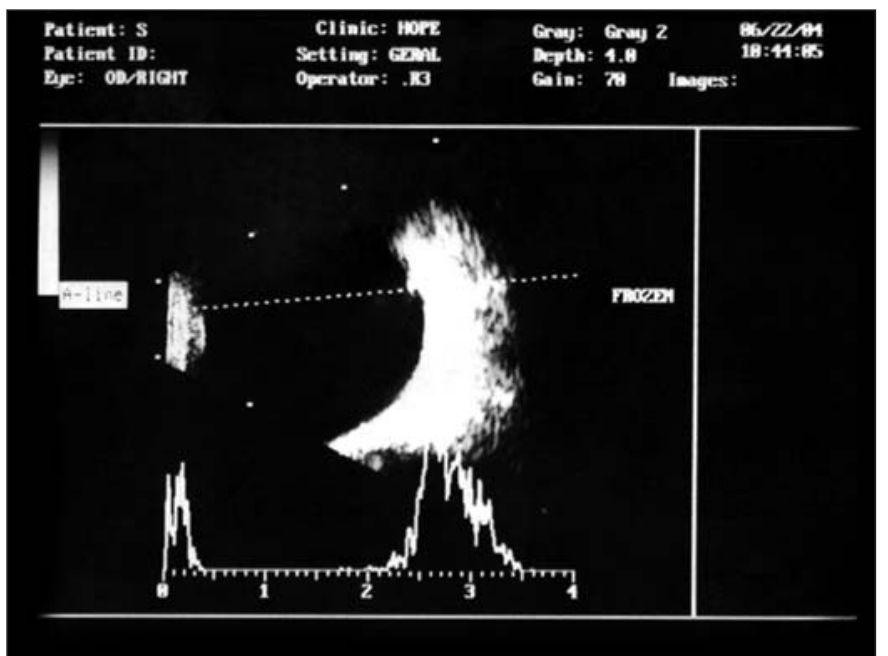

Figura 8 - Ultra-sonografia ocular A e B scan - Eco membranáceo de baixa refletividade em cavidade vítrea, em contato com a parede posterior do globo ocular

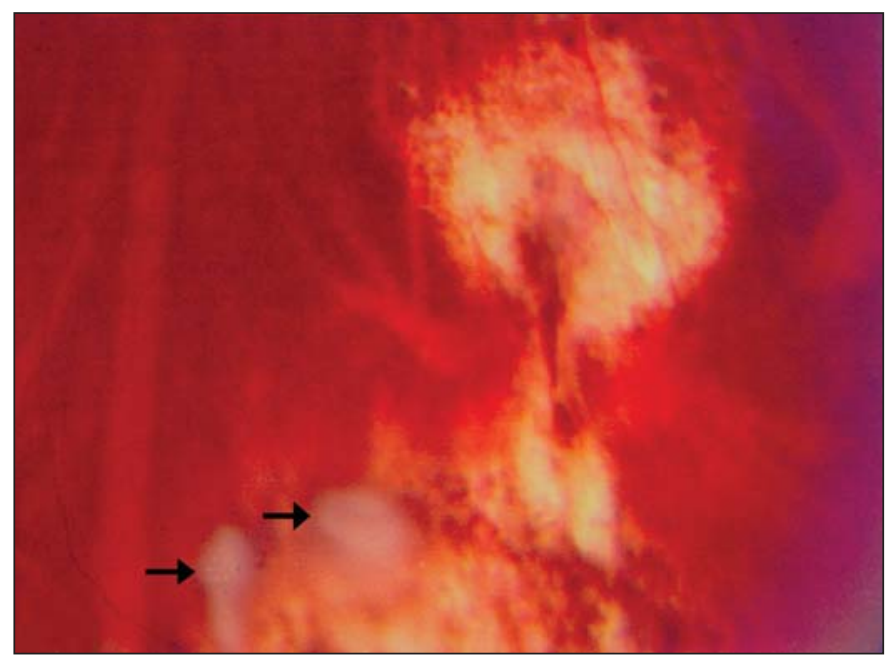

Figura 9 - Retinografia após braquiterapia com lodo ${ }^{125}$ e tratamento local (crioterapia e TTT). Ampliação de área atrófica do EPR e presença de duas condensações na borda da lesão.

retina, no intuito de observar o comportamento desta lesão (Figura 10). Houve modificação da forma da condensação, que se tornou mais alongada e esta se depositou na superfície da retina nasal equatorial (Figura 11), sendo tratada com TTT.

Com seguimento total de 38 meses após o aparecimento do tumor bilateral, não se pode afirmar uma condição de estabilidade do quadro clínico da neoplasia até o presente momento e mesmo estando o paciente com boa acuidade visual e exames de controle sistêmicos normais, o caso requer um controle rigoroso diante da sua evolução.

\section{DISCUSSÃO}

O retinoblastoma é um tumor intra-ocular caracteristicamente da infância e acredita-se ser originado de células pre-

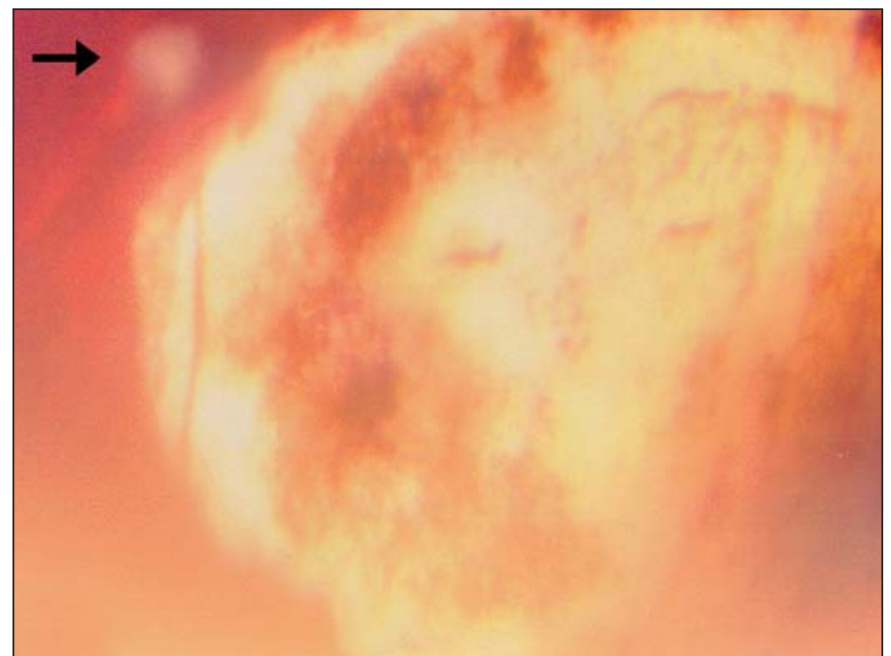

Figura 10 - Retinografia de controle após 6 meses do último tratamento - Nova condensação flutuante em cavidade vítrea, com menos que $1 \mathrm{DD}$, próxima à lesão atrófica, esta sem sinais de atividade tumoral aparente

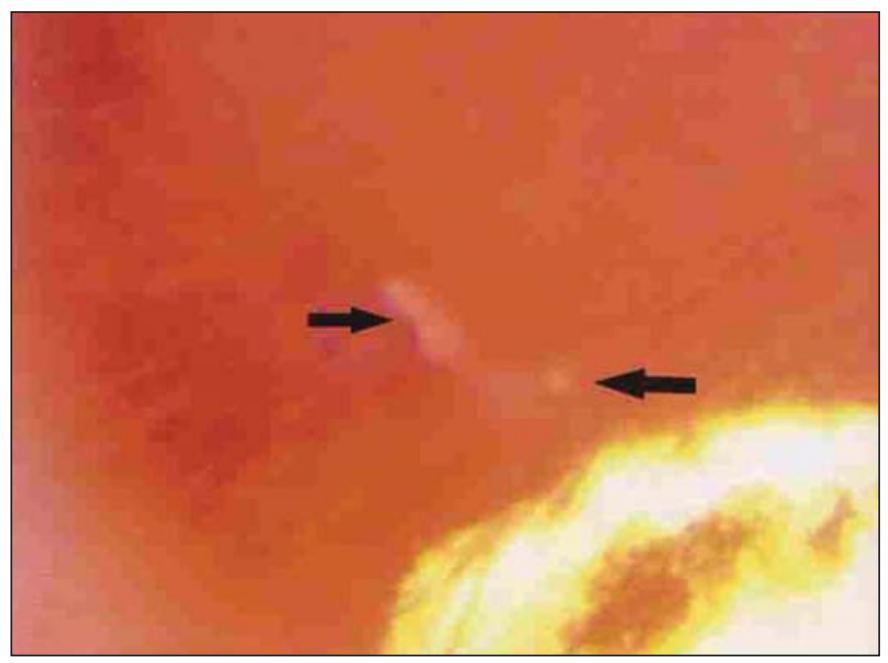

Figura 11 - Retinografia. Após 15 dias de controle, a mesma condensação flutuante em cavidade vítrea, modificou-se em forma e subdividiu-se, sem haver depósito na superfície da retina

cursoras dos fotorreceptores da retina. ${ }^{(3)}$ Pacientes com história familiar positiva têm seu diagnóstico mais precoce, algumas vezes dentro dos primeiros dias de vida. Em casos com história familiar negativa, unilateral ou bilateral, o diagnóstico em geral se dá mais tardiamente ${ }^{(5)}$.

Alguns autores revisaram 400 casos de retinoblastoma, onde $8,5 \%$ eram crianças maiores de cinco anos de idade. Apenas $0,8 \%$ tinham mais de 15 anos ao diagnóstico inicial ${ }^{(4)}$.

A incidência de tumores bilaterais diminui após dois anos de idade com picos entre quatro e cinco meses e entre os unilaterais, o pico ocorre entre seis e sete $\operatorname{meses}^{(2)}$.

As características clínicas da criança demonstram apresentação atípica no que concerne à idade do aparecimento da bilateralidade do tumor, assim como a evolução clínica antes e após tratamento. 
Tem sido relatado início tardio dos sintomas de casos de retinoblastoma em adultos ${ }^{(5)}$. Na avaliação do presente caso, o paciente teve o diagnóstico de retinoblastoma unilateral aos nove meses e em seu controle, durante 10 anos, acreditou-se que o mesmo estivesse fora de risco para a bilateralidade, mesmo com história familiar positiva. Esse intervalo do aparecimento do tumor bilateral é pouco freqüente, mesmo em outros relatos da literatura. Nos casos de apresentação tardia são postuladas duas teorias. Na primeira, é postulada a transformação maligna de células embrionárias da retina ${ }^{(8)}$, e a outra, a reativação de retinoblastoma de regressão espontânea ${ }^{(9)}$.

Embora o retinoblastoma seja incomum antes dos 10 anos de idade, a apresentação em grupo de idade mais avançada pode existir, havendo descrições em adulto de $74 \operatorname{anos}^{(10)}$. No presente relato, no exame inicial e em todos os outros que se seguiram durante o acompanhamento deste paciente, não havia lesão no olho contralateral, o que se leva a crer na possibilidade de algum fator desencadeante para a ativação dessas células retinianas imaturas. Não se sabe se estímulos hormonais poderiam estar envolvidos nessa cadeia de ativação, já que este aparecimento se deu durante a fase puberal do paciente, o que estimula investigação mais aprofundada.

Embora o retinoblastoma apresente-se, geralmente, com quadro clínico clássico, incluindo leucocoria e estrabismo, outros sinais da doença, entretanto, podem ocorrer, como rubeosis $^{(11)}$, hifema e hemorragia vítrea. A forma oligossintomática com a qual se apresentou o paciente do presente relato reafirma a necessidade de manutenção de acompanhamento periódico do olho contralateral em longo prazo, mesmo naqueles casos em que se considera cura após enucleaçâo do primeiro olho.

A abordagem terapêutica, usando-se tratamento local com termoterapia transpupilar, e, posteriormente, braquiterapia com Iodo ${ }^{125}$, resultou em regressão parcial da neoplasia, contudo, houve o aparecimento de outras sementes no seguimento clínico, demonstrando alta agressividade do tumor. Não foi indicado esquema de quimioterapia associada ao tratamento local, pois, no seguimento, a lesão encontrava-se completamente atrófica, sem vascularização ou área tumoral residual. A possibilidade de irradiação da órbita por radioterapia externa conformacional em três dimensões foi aventada por ocasião do aparecimento da última condensação vítrea; mas por ter havido depósito na superfície da retina, optou-se por tratamento local com TTT.

A característica da evolução do caso relatado levanta a possibilidade de se estar diante de um tumor germinal, com forte penetrância e comportamento agressivo, de difícil controle, onde possivelmente a abordagem do tratamento através de terapia gênica ${ }^{(12)}$ poderia ser uma opção mais adequada. Por outro lado, a apresentação atípica e tardia do paciente relatado pode constituir a base para maiores investigações na condução desses tumores.

Como perspectiva futura as pesquisas utilizando imunohistoquímica para dosagem da enzima ácido graxo sintetase ${ }^{(13)}$, presente em diversos tipos de câncer, inclusive no retinoblas- toma, poderão relacioná-la às apresentações clínicas de caráter mais agressivo, podendo fornecer dados promissores para seguimento e avanços terapêuticos.

\section{ABSTRACT}

A case of retinoblastoma with uncommon features is reported, aiming at improving follow-up. In 1993, SJMMF, 9-month-old white boy, presented a squint in the left eye. A retinal tumor was detected. The patient had a family history of retinoblastoma. Enucleation was performed and retinoblastoma was proved. The patient underwent examination for staging, all were normal. The fellow eye remained normal for 10 years. During routine examination the retina map revealed three retinoblastoma white lesions in the nasal retina. The patient underwent transpupillary thermotherapy with diode laser. After 30 days, the lesions became atrophic. After 60 days there was tumor recurrence on the border of the lesion. Sclera cryotherapy was performed. There was tumor regression for six months. During follow-up, condensations next to the atrophic tumor lesions were discovered, which were vitreous seeds. Brachitherapy with 125 iodine was done. The lesions disappeared after 30 days. New vitreous seeds appeared 3 months later, set on the retina surface. They were blocked with sclera cryotherapy and transpupillary thermotherapy. The patient presented with new vitreous seeds after six months, which adhered to the retina. They were blocked with transpupillary thermotherapy. Follow-up was for 38 months since the appearance of the bilateral tumor. The patient presents normal visual acuity and clinical examination. This case is important considering the low frequency of the disease at this age. It is essential to maintain alertness when cases of retinoblastoma are seen as cured.

Keywords: Retinoblastoma/epidemiology; Eye enucleation; Laser; Induced hyperthermia; Humans; Male; Infant; Case reports [Publication type]

\section{REFERÊNCIAS}

1. Shields CL, Shields LL. Retinoblastoma. Rev Soc Bras Retina Vítreo. 2004; 8:10-5.

2. Young Jr JL, Smith MA, Roffers,SD, Liff JM, Bunin GR. Retinoblastoma [Internet]. Bethesda, MD: National Cancer Institute; 1995. p.73-7. [Seer Pediatric Monograph] [cited 2007 Sept 15]. Available from: http://seer.cancer. gov/publications/childhood/retinoblastoma.pdf

3. Abreu AA, Ventura LO, Abreu SS, Regis L, Morais V, Calheiros LM. Epidemiologic study of retinoblastoma in Recife - Pernambuco - Brasil: january 1985 - July 1997. Arq Bras Oftalmol.1999;62(5):614-5, 618-9.

4. Shields CL, Shields JA, Shah P. Retinoblastoma in older children. Ophthalmology. 1991;98(3):395-9.

5. Mietz H, Hutton WL, Font RL. Unilateral retinoblastoma in an adult: report of a case and review of 1iterature. Ophthalmology. 1997;104(1):43-7. Comment in: Ophthalmology. 1997;104(8):1207; Ophthalmology. 1997;104(11):1728.

6. Rodriguez Rodrigues BN, Vigoa Aranguren L. Retinoblastoma atípico: a propósito de un caso. Rev Cuba Oftalmol [Internet]. 2003 [citado 2007 Mar 18];16(1): [3 telas]. Disponible en: http://bvs.sld.cu/revistas/oft/vol16_01_03/ oft08103.htm 
7. Park JJ, Gole GA, Finnigan S, Vandeleur K. Late presentantion of a unilateral sporadic retinoblastoma in a 16-year-old girl. Aust N Z J Ophthalmol. 1999; 27(5):365-8.

8. Takahashi T, Tamura S, Inoue M, Isayama Y, Sashikata T. Retinoblastoma in a 26 year-old adult. Ophthalmology.1983;90(2):179-83.

9. Lash H. Ein retinoblastomrezidiv im erwachsenenalter. Klin Monastsbl Augenheilkd. 1964;144:268-72.

10. Findlay JR, Byron H. Retinoblastoma in adult: review of literature and report of case associated with benign melanoma. Acta XIX Concilium Ophthamologicum, New Delhi. 1962;2:1168-78.
11. Pe'er J, Neufeld M, Baras M, Gnessin H, Itin A, Keshet E. Rubeosis iridis in retinoblastoma. Histologic findings and the possible role of vascular endothelial growth factor in its induction. Ophthalmology. 1997;104(8):1251-8.

12. Harbour JW. Molecular therapy for retinoblastoma [Abstract]. In: $30^{\text {th }}$ Biannual Meeting of International Society for Genetic Eye Disease (ISGED); $10^{\text {th }}$ International Symposium on Retinoblastoma. 2001; Fort Lauderdale, Florida; 2001.

13. Camassei FD, Cozza R, Acquaviva A, Jenkner A, Ravà L, Gareri R, et al. Expression of the lipogenic enzyme fatty acid synthase (FAS) in retinoblastoma and its correlation with tumor aggressiveness. Invest Ophthalmol Vis Sci. 2003;44(6):2399-403. 